\title{
Aligning positioning and Navigation of smart mobile devices to AR/VR environments
}

\author{
Kaimaris, D. ${ }^{1}$, Roustanis, Th. ${ }^{2}$, Karolos, I. A. ${ }^{2}$, Klimantakis, K. ${ }^{3}$, Patias, P. ${ }^{*}$ \\ ${ }^{1}$ School of Spatial Planning and Development, Aristotle University of Thessaloniki, Greece - kaimaris@auth.gr \\ ${ }^{2}$ School of Rural and Surveying Engineering, Aristotle University of Thessaloniki, Greece - (throu, ikarolos, patias)@auth.gr \\ ${ }^{3}$ Department of Applied Informatics, University of Macedonia, Greece - dai16010@edu.uom.gr
}

Keywords: Augmented Reality (AR), Smart mobile devices, Wi-Fi Fingerprinting, UWB, BLE, GNSS, Cultural Heritage

\begin{abstract}
:
Augmented Reality/Virtual Reality (AR/VR) technology offers novel and excited experiences to visitors of cultural sites. Extended recent technological advances in smart communication devices made the connection of the real to the virtual worlds more affordable and effective. In spite of the rapid development and availability of the AR/VR applications to cultural heritage, there still exist gaps and challenges in accurate positioning and navigation of visitors' smart devices in both out-doors archaeological spaces as well as in in-doors museum spaces. Recent technology smart devices embody GNSS positioning sensors, which in best cases, provide 1-meter positioning accuracies in open spaces, while are unable to work in-doors. Such accuracies and restrictions lead to gaps in fetching visitor's position in AR environments and mis-positions with regard to the rest AR/VR objects as visualized in smart screens, while the problem gets worse when dealing with real-time videoing, when elapse times and refresh rates lead to additional mispositionings. In this paper, effort is made to present and evaluate the most recent and widely used tools for positioning and navigation of smart devices in in- and out-doors $\mathrm{CH}$ sites.
\end{abstract}

\section{INTRODUCTION - METHODS AND CHALLENGES OF REPRESENTATION OF AR POINTS IN THE PHYSICAL WORLD}

To meet up the expectations of visitors, cultural heritage sites are always looking for different and innovative approaches. For that reason, many places with cultural interest are enriched by specially adapted to their exhibits augmented reality (AR) applications, facilitating the understanding of their individual elements.

This enrichment through the interconnection of the real with the virtual world, is economically and temporally more affordable than ever, as in most cases no installation of complex equipment is required in the respective museum or archaeological site. And that because, the development of smart mobile devices (Smartphones, Tablets) is rapid and at the same time their adoption by the consumers, part of which visits cultural heritage sites. According to (Haugstvedt et al. 2012), mobile augmented reality (MAR), is one of the fastest growing research areas in the field of mixed reality technologies (MR).

Most of the smart devices produced today have upgraded technical features, suitable to support AR applications. Some of them are the fast processors, multiple cameras systems, Time of Flight (TOF) lenses for depth recognition, Inertial Measurement Units (IMUs) in conjunction with Global Navigation Satellite Systems (GNSS), high-brightness and high-definition touch screens as well as super-fast interconnection and networking technologies such as $4 \mathrm{G}, 5 \mathrm{G}$, Wi-Fi 6 , Bluetooth 5 etc. In addition to the above electronic parts, smart mobile devices are accompanied by advanced operating systems (iOS, iPadOS, Android, etc.) on which have been developed multiple programming tools - Software Developer Kits (SDKs), which facilitate and automate the development processes of the AR applications. These tools allow virtual reality application developers to determine in real time the location of the user's mobile device camera, while simultaneously displaying virtual 3D models on desired locations and surfaces in the real world.
Google Inc. ARCore, Apple Inc. ARKit, and Vuforia Engine for Unity are just a few examples of virtual reality programming tools. These programming tools include the following three distinct algorithmic procedures:

Motion tracking: As the user moves the smart device, a process known in the literature as visual inertial odometry (Chang, L. el al. 2019) is applied. This process is based on the detection of distinctive feature points on each image recorded by the camera of the mobile device. The exported visual information is combined with inertial and location measurements from the device's IMU and GNSS sensors to assess the position and orientation (pose) of its camera system, relative to the physical world and in real time.

Environmental understanding: This process involves grouping the characteristic points, which are identified by computer vision algorithms, and belong to common horizontal or vertical surfaces (e.g., tables or walls). The recognized surfaces from this process, are then defined as planes with distinct boundaries on which virtual 3D models can be placed, making them look like part of the real world (Feigl, Tobias et al. 2020).

Depth understanding: This process creates depth maps, or images that contain data about the distances of surfaces in relation to a given point, using the central RGB camera of the mobile device. In fact, in some mobile devices that contain TOF lenses, there is the possibility of scanning the surfaces and objects of the physical space and extracting point clouds in real time. The process of depth understanding, among others, allows the correct placement of virtual models on the front or back of the physical world's objects (Herbers, Patrick el al. 2019).

The above algorithmic procedures offer to the smart mobile devices users, satisfying results in small-scale augmentation spaces, with distinct surfaces and textures. However, challenges are found in larger-scale areas where users encounter complex surfaces without characteristic texture and distinct patterns and with demanding lighting conditions, such as outdoor archeological sites and museums. In these areas, locating the position and orientation of the camera of the mobile device,

\footnotetext{
* Corresponding author
} 
contains errors related to the accuracy of the integrated to the smart devices, GNSS sensors, as well as the lack of the ability of the vision algorithms to detect the so-called characteristic points in the physical world. GNSS receivers in most smart mobile devices have a positioning accuracy that does not exceed 2-5 meters outdoors without high distinct obstacles (e.g., trees, buildings, etc.), while on indoors they cannot provide any measurement information. This paper identifies the different solutions and the accuracy limits of the available positioning technics on mobile devices in AR applications. Specifically, in section 2 that follows, the most common solutions for locating mobile devices indoors and outdoors, which can be adapted to smart mobile devices and used in AR applications of cultural interest, are explored, while section 3 evaluates the position accuracy offered. In conclusion the advantages and disadvantages of each positioning technology are presented.

\section{INDOOR AND OUTDOOR LOCATION TRACKING TECHNOLOGIES}

Finding the user's location with optimal accuracy is a basic need for the proper representation of AR content both for outdoors and indoors environment. The most popular positioning technology in smart mobile devices is based on the Global Navigation Satellite Systems (GNSS). The United States Global Positioning System, GPS (GPS 2021), the Russian GLONASS (GLONASS 2021), the European Galileo (Galileo 2021) and the Chinese BeiDou (Beidou 2021) are the most widely used and supported by all smartphones devices on the market. At the same time, selected devices are supporting dual carrier frequency GNSS (L1, L5), ensuring better positioning accuracy in difficult conditions of signal propagation, such as in locations surrounded by tall buildings and obstacles, where the signal multipath error is introduced (Vasisht, D, et al. 2016). Despite the above additions of technologies to the modern mobile devices, positioning accuracy through the built-in GNSS systems, is limited to one meter ( $1 \mathrm{~m})$ in real time (GPS 2021), while this performance is for outdoor use only. Instead, to locate the user's location in an indoor environment, alternative wireless technologies are used, available on a variety of mobile devices through appropriate fixed signal sources or access points (Access Points - APs). In an indoor localization system, there are two options for someone to find access points. APs that are already installed indoors such as Wi-Fi routers, or APs that are established from the installation of appropriated beacons based on Bluetooth Low Energy (BLE) and Ultra-Wide Band (UWB) protocols. As the internal environments such as museums are complex (multiple rooms, floors, and load-bearing masonry), multipath and shadowing errors of radio signals are introduced in this case as well (Liu, H. et al. 2011). Therefore, the received signal may include line of sight (LOS) and non-line of sight (NLOS) effects (Xiong, J, et al. 2015). The accuracy and measurement techniques of indoor and outdoor positioning technologies are examined below.

\subsection{UWB sensor technology and manufacturers}

UWB technology uses very low energy to transmit high volumes of data over short distances over a large portion of the radio spectrum. A radio wave emitted is generally considered UWB if its bandwidth exceeds $500 \mathrm{MHz}$ or $20 \%$ of the carrier frequency (Luo, Y. et al. 2012). The properties of UWB protocol such as relatively low power consumption, efficient signal penetration into dense materials and reduced multireflection on metal surfaces, make it suitable for use in indoor positioning systems. At the time of writing, Decawave is the leading manufacturer of UWB sensors for indoor positioning.
The mobile beacons produced by the company Decawave, can operate with simple low capacity (220 mAh) coin cell type batteries for up to a few months with just one charge (DWM1001 2021) under suitable conditions, while they can send their location data via Bluetooth in the augmented reality system with an accuracy of $0.1-0.2 \mathrm{~m}$ horizontally and vertically. The extraction of the user's location on the Decawave's mobile beacons (Tags), requires the installation of corresponding fixed beacons (Anchors) in places with wellknown coordinates (Figure 1) with respect to the reference system of the indoor installation site (e.g., museum or indoor archeological site). The fixed Anchors radio beacons should be powered by a continuous power supply and not by a battery. In addition to the Decawave system which requires the user to bring an additional device, the UWB protocol seems to be integrated into a portion of smart mobile devices.

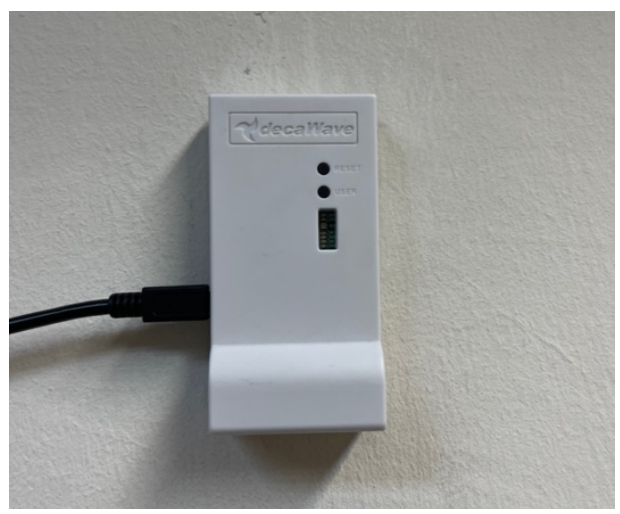

Figure 1: Decawave's UWB Anchor Beacon

Apple and Samsung are now incorporating into their latest models, iPhone 11, 12 series and Galaxy Note 20 Ultra, S21 Ultra respectively, chipsets compatible with the UWB protocol, but without providing a complete SDK to application developers, intended for indoor localization. For the time being, Apple is providing its developers with the Nearby Interaction Framework tool, which can calculate the distance between two close compatibles with UWB technology iPhone, via the builtin U1 chip, with centimeter level of accuracy. In addition, both Apple and Samsung produce individual UWB mobile sensors (Airtags and Smart Tags respectively) designed solely to find lost items with the help of UWB compatible mobile devices at close range and not to implement them as fixed anchors with absolutely known coordinates that could help to develop an indoor positioning system like Decawave one.

\subsubsection{Signal measurement and localization techniques with the UWB protocol}

The two most common techniques for measuring the distance between fixed (Anchors) and portable (Tags) UWB beacons are Time of Arrival (TOA) and Time Difference of Arrival (TDOA).

In the TOA technique, the travel time, or flight time, that a radio signal travels from the transmitter to the receiver, moving with the speed of light, is calculated. Accurate synchronization between fixed and mobile radio beacon is required to measure the above time. The TOA technique is described by Equation 1:

$$
d_{i}=\left(t_{i}-t_{0}\right) * c
$$

where:

$c=3 * 10^{8} \mathrm{~m} / \mathrm{s}^{2}$ is the speed of light, 
$t_{0}, t_{i}$ is the time of transmission and reception of the signal respectively,

$d_{i}$ is the final distance between the beacon with perfectly known coordinates (Anchor or AP) and the user's moving beacon (Tag).

The coordinates of the mobile receiver carried by the user (Tag) are then calculated based on the distances extracted from the TOA process in relation to three at least fixed Anchor Beacons with perfectly known coordinates, through the Trilateration technique and by applying a system of least squares (least squares system), as shown in Figure 2 (Waadt, A. et al 2020).

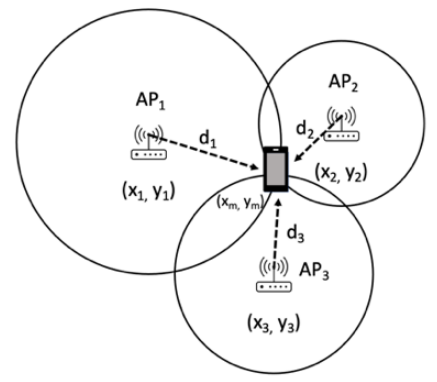

Figure 2: Finding a location via UWB using the TOA and Trilateration technique

A variation of the TOA method, the Two Way Ranging (TWR) method in conjunction with the application of the Trilateration algorithm, is the default process for extracting user coordinates contained in the Decawave beacon firmware. In the TWR method, the mobile beacon sends a radio signal to the fixed beacon and records the transmission start time $\left(t_{1}\right)$. The fixed beacon receives the signal and in turn transmits a response (radio signal) back to the mobile (Tag) with a time delay $\left(t_{\text {reply }}\right)$. The mobile beacon receives this response and records the reception time $\left(t_{2}\right)$. The final distance $\left(d_{i}\right)$ between the fixed and the mobile beacon is given by Equation 2 considering that the radio signals move with the speed of light $(c)$ :

$$
d_{i}=\frac{\left(t_{2}-t_{1}-t_{\text {reply }}\right)}{2} * c
$$

The TWR technique has the advantage that it does not require clock synchronization between Tag and Anchor beacon and the disadvantage that is reducing the battery life of the mobile beacon. Based on the TWR technique with $1 \mathrm{~Hz}$ refresh rate and with the help of small 16340 rechargeable batteries, the Decawave Tags can be operated for at least 24 hours of continuous use, without intermediate charging (DWM1001 2021)

The TDOA technique is based on the difference in reception time of a radio signal from the fixed beacons, emitted by the user's mobile beacon (Figure 3).

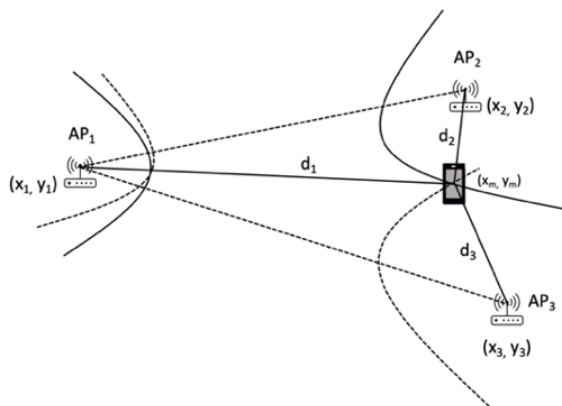

Figure 3: Finding a location via UWB using the TDOA technique
The following Equation 3, calculates the distance between the fixed Anchor to which the radio signal of the mobile Tag first reaches in relation to the other Anchors of the UWB indoor location system:

$$
\begin{aligned}
d_{i j}=\left(t_{i}-t_{j}\right) * c & =\sqrt{\left(x_{i}-x_{m}\right)^{2}+\left(y_{i}-y_{m}\right)^{2}} \\
& -\sqrt{\left(x_{j}-x_{m}\right)^{2}+\left(y_{j}-y_{m}\right)^{2}}
\end{aligned}
$$

where: $t_{i}, t_{j}$, is the time that the radio signal was received by Anchors $i$ and $j$ respectively,

$c$ is the speed of light,

$\left(x_{i}, y_{i}\right),\left(x_{j}, y_{j}\right),\left(x_{m}, y_{m}\right)$ are the coordinates of Anchor $i$ and $j$ and the moving Tag, respectively.

Geometrically, the final position of the Tag mobile beacon is at the intersection of the anchors-centric hyperbolas, as shown in Figure 2. Unlike the Decawave TWR technique, the TDOA technique requires complete synchronization of the clocks of the fixed Anchors participating in the location system. This results in a more complex installation process as it is often required to connect the fixed Anchors to a central location synchronization and output system via cables (Witrisal, K. et al. 2016). However, this synchronization ensures the moving beacons, lower energy consumption (up to a few months with a charge) as it is enough to transmit the radio signal and receive it from the APs. The TDOA technique can also be implemented by specific Decawave beacons at a higher cost, however, many times higher than the TWR beacons.

\subsection{Positioning technology with $\mathrm{Wi}-\mathrm{Fi}$}

Wi-Fi protocol is a technology of local wireless networking (WLAN), devices based on the 802.11 IEEE network standard that operates in the 2.4 and $5 \mathrm{GHz}$ radio frequency bands. All smart devices, such as Smartphones and Tablets, support Wi-Fi to meet the needs of wireless connectivity and data exchange indoors and outdoors. The most important advantage of Wi-Fi technology in the implementation of an indoor location system, is the ease of installation. Most building facilities already have a plethora of Wi-Fi APs in multiple locations for internet connectivity, which, combined with users' smart devices, are capable of hosting a real-time location system. However, because the primary role of $\mathrm{Wi}-\mathrm{Fi}$ is not the location finding, it requires the use of specialized algorithmic processes that must be implemented and supported by users' mobile devices. The method of measuring RSS signals and calculating the user's location through the Fingerprinting process are the main algorithmic procedures applied through appropriate SDKs (such as the Indoor SDK of Here Maps etc.) to users' mobile phones and provided to application developers for the implementation of indoor location systems. The above programming tools, which make use of Wi-Fi technology, are available at the time only on Android operating system. iOS, iPadOS does not provide an available API. However, Apple allows closed public spaces (as well as places of cultural interest) to submit an application to the Indoor Map Program (Apple Indoor Map Program 2021) in order to map their area through a suitable Fingerprinting application provided to them. In case of approval of this application, the process of extracting the user's location in the area of interest is done only through the application of the Apple Maps. The above procedure is in no way appropriate for extracting metering information to support $\mathrm{AR}$ applications in areas of cultural interest. Recently, restrictions have been introduced on the use of Wi-Fi technology for location finding in Android environments, which also concern the frequency of scanning Wi-Fi signals from the mobile device. These 
restrictions are introduced for the first time in Android version 8 (API level 26) and continue until the latest version 11 (API Level 30). These limitations can be overcome on a portion of Android mobile devices if the user turns off an appropriate setting (Wi-Fi scan throttling) through the Developer Options menu of their mobile device. The accuracy that someone can expect from Wi-Fi technology for indoor localization is a few meters ( 2 meters and above).

\subsubsection{Signal measurement and localization techniques with Wi-Fi protocol}

Wi-Fi signal analysis is done via RSS feeds. RSS is a measure of the strength of a received radio signal. RSS is measured in decibels-milliwatt $(\mathrm{dBm})$ and takes negative values ranging from $0 \mathrm{dBm}$ (high power signal) to $-100 \mathrm{dBm}$ (very low power signal). As the distance between the Wi-Fi AP and the user's mobile device increases, the RSS value weakens due to multiple factors such as the antenna of the transmitting and receiving devices, the type of masonry and the surrounding floors, the number of people inside the indoor space, etc. It should be noted that the RSS value does not decrease linearly as the distance increases (Anagnostopoulos, G. et al. 2014). RSS modeling is usually performed through a combination of largescale and small-scale attenuation effects (Sklar, B et al. 1997). The large-scale attenuation component depicts the attenuation of the radio signal, when it crosses for example a dense masonry or a floor until it ends up in the user's smartphone. The average RSS value is derived from the large-scale component and usually follows the normal distribution (Kaemarungsi, K. 2005). Similarly, the small-scale attenuation component describes the RSS fluctuation due to reflection error and follows the Rayleigh distribution (Jianyong, Z et al. 2014). Finally, RSS value fluctuations are filtered through the Gaussian filter.

The moving user's coordinates extraction in an internal location finder system based on the Wi-Fi protocol is done through the Fingerprinting technique. In the Fingerprinting technique, the RSS values of neighboring Wi-Fi APs are measured at various reference points and stored together with the corresponding well-known coordinates of the points in a database. In the process of locating a user, the new RSS feeds of the transmitted Wi-Fi signals are measured and compared with those stored in the database to evaluate a new location. Therefore, as shown in Figure 3, the implementation of the Fingerprinting technique includes two distinct phases, the data collection phase (offline phase) and the phase of finding the user location (online phase) (Kanaris, L. et al. 2017). In the data collection phase, the internal reference space in which the positioning system will be implemented is divided into a grid of $1 \mathrm{x} 1 \mathrm{~m}$ points. A mobile device goes over each point of the grid, recording all the RSS values of the neighboring $\mathrm{Wi}-\mathrm{Fi}$ in correspondence with the exact coordinates of the point with respect to the indoor reference system. In the locating phase, the user moves indoors, steadily scanning the transmitted RSS feeds and comparing them to those stored in the database during the data collection phase to extract their final location (Figure 4).

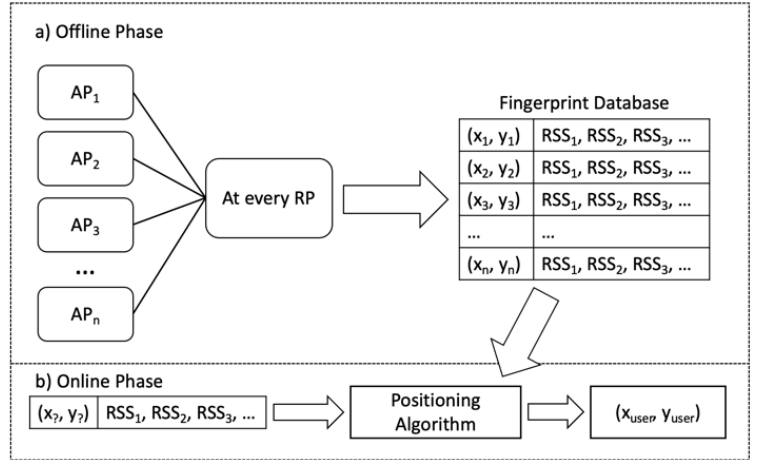

Figure 4: The process of installation and implementation of the Fingerprinting method

The process of locating Fingerprinting is characterized by the time-consuming phase of data collection in large buildings, while offering good accuracy during the phase of locating the user $(2 \mathrm{~m})$.

\subsection{Technology and manufacturers of BLE sensors for positioning}

The BLE protocol first debuted in June 2010 with Bluetooth 4.0. It is designed for the wireless transmission of small volumes of data between the devices that support it, in a short range and with low battery consumption (Kriz, P. et al. 2016). By comparison, BLE technology consumes less power compared to Wi-Fi technology. Similar to Wi-Fi, BLE operates in the ISM $2.4 \mathrm{GHz}$ radio band. The BLE radio frequency band is divided into 40 channels, the distance between which is set at $2 \mathrm{MHz}$ (Spachos, P. et al. 2020). Channels 37, 38 and 39 are the main data transmission channels while the rest are the secondary ones. The three main channels have been strategically placed to avoid signal interference with other common protocols, such as IEEE 802.11 and ZigBee (Corbacho S. 2014). A BLE radio beacon can transmit radio frequencies per 100 to $2000 \mathrm{~ms}$. For the needs of implementing an indoor positioning system, this rate is usually adjusted to $300 \mathrm{~ms}$, since the average walking rate is $1.3 \mathrm{~m} / \mathrm{s}$. Unlike Wi-Fi, the BLE scanning speed of radio signals is not limited so that the position of a mobile user can be calculated with a frequency of up to $1 \mathrm{~Hz}$.

BLE technology is supported by both Android and iOS/iPadOS operating systems. However, the compatibility of an application to be developed may differ depending on the available BLE (Eddystone and iBeacon) data transfer protocols. The Eddystone protocol is open source and developed by Google, while it is compatible with Android and iOS devices. In contrast, the iBeacon protocol was developed by Apple and is only compatible with Apple devices. In the Eddystone protocol, the three main BLE channels transmit a unique identifier (Eddystone-UID), a compressed web link (Eddystone-URL), and telemetry data (Eddystone-TLM), respectively. In the iBeacon protocol we have the three transmission elements UUID, major and minor respectively.

In BLE technology the distance is exported as in Wi-Fi technology, by measuring the RSS values between the fixed BLE Beacons and the smart mobile devices of the users who move inside an interior. In the BLE protocol, RSS values can be transformed into distances through the following Equation 4:

$$
d=10^{(P-R S S I) /(10 * n)}
$$

where: 
$d$ is the final distance between the fixed and the mobile BLE Beacon,

$P$ is the RSS value we receive if we place the user's mobile device one meter away from the fixed BLE Beacon,

RSSI is the RSS value at the receiving point of the BLE signal at which we want to measure the distance relative to the fixed Beacon,

$n$ is a signal propagation constant that takes values between 2-4 and defines the propagation environment ( 2 in open environment, 4 in environment with several obstacles).

After extracting at least three distances from fixed BLE beacons with well-known coordinates, the Trilateration technique is applied (as in the case of UWB) for the final calculation of the coordinates of the user's mobile device. In addition to the Trilateration technique, in an internal positioning system with BLE beacons, we can use the Fingerprinting technique in the same way as in the case of Wi-Fi. Based on the above processes, the positioning accuracy in the BLE technique ranges from 1-2 m

There are a number of BLE beacon manufacturers on the market (eg Estimote, Minew, kontakt.io, etc.) which, in addition to hardware, also provide SDKs for application developers in order to extract the position of users entering an area of interest BLE radio beacons are in most cases powered by coin cell batteries, while their lifespan (both in fixed to known coordinates and in portable ones) ranges from 2 to 5 years, depending on the rate of transmission of the transmitted information (advertising packets).

\subsection{Determination of outdoor localization using GNSS systems}

Global Navigation Satellite Systems (GNSS) can provide the $3 \mathrm{D}$ geodetic coordinates of the measured points of the physical environment in real time (Fotiou, Pikridas 2012). When incorporating related assay techniques, such as the Real-Time Kinematic (RTK) method (Rietdorf et al., 2006) which combines phase measurements of radio signals with corrections from permanent reference stations with well-known coordinates, the GNSS systems is able to achieve up to one centimeter accuracy in real-time in location. extraction. For several years, such GNSS receivers were intended exclusively for use in high-precision geodetic applications, due to their increased purchase cost and size. In recent years, however, high-precision GNSS systems can be installed on a variety of smart mobile devices, either internally in SOC (System on a Chip) format, or externally, communicating wirelessly or wired with multiple communication protocols (Bluetooth, Wi-Fi, USB etc.).

With the advent of Android 10 (API level 29), smart mobile devices can now export and process code and phase measurements. It is worth noting that at the time of writing, approximately $84 \%$ of Android devices support the GNSS metrics export API from their built-in chipset. (GNSS RAW Data Measurements). At the same time, 2018 marked the arrival of the first Android Smartphone (Xiaomi Mi 8) with dual frequency GNSS support (L1/L5). Since then, multiple Android smartphones have been released with the above feature, on which the best ionosphere and multipath error reduction technics are based. So, with the help of the above parameters on selected Android devices and by applying relevant positioning techniques we can now extract the user's location, with an accuracy of one meter or better.

At the same time, the accuracy of low-cost receivers that can be adjusted with appropriate communication protocols on the outer casing of the smartphones is in the order of a few centimeters in real time. An example of an externally customizable receiver is the u-blox receiver, ZED-F9P (Figure 5), which incorporates a high data rate and has the appropriate RTCM data processing software to implement the RTK method (Fotiou, Pikridas 2012).

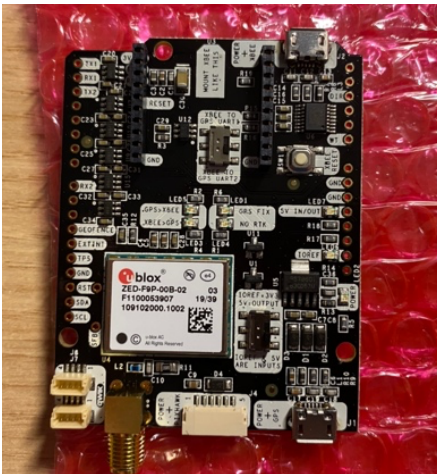

Figure 5: The external u-blox ZED-F9P GNSS module

\section{CONCLUSIONS, COMPARISON OF POSITIONIMG METHODS, POSITION ACCURACY, ADVANTAGES AND DISADVANTAGES}

Based on the above analysis of location technologies, the following conclusions are drawn as to their suitability for use in AR applications, for indoors or outdoors cultural heritage sites.

The UWB protocol, is the most appropriate technology for indoor localization, utilizing important advantages such as good reliability of measuring distances between beacons which are not greatly affected by obstacles and metal surfaces, threedimensional accuracy of $20 \mathrm{~cm}$ in real-time and low purchasing cost of the TWR sensors and accessories. The main disadvantages of UWB technology are the need to install additional fixed (Anchors) beacons with permanent power supply, in locations with known coordinates and at different points up to 50 meters apart, within the closed space of the internal positioning system. An additional disadvantage is that the users need to transfer a mobile (Tag) UWB radio beacon that is extracting their location to their smart devices via BLE protocol technology. Especially in the TWR method, a parallel disadvantage is the relatively low battery life of the mobile beacons, which for the Decawave solution is calculated in a period of one month. The obligation to carry additional UWB sensors with limited battery life by the user, however, is expected to be eliminated in the coming years, as already three of the largest manufacturers of smart devices (Apple, Samsung and Xiaomi) are installing UWB sensors inside their devices in selected models.

Wi-Fi is generally considered unsuitable for use in locationfinding processes intended for AR applications. This is due to the reduced positioning accuracy it offers (over $2 \mathrm{~m}$ ) and the lack of support for software development tools for iOS/iPadOS. Lack of compatibility is also found in various models of mobile devices that work based on the version of Android 8 (API level 26 ) or later. An indoor location system based on the Wi-Fi protocol, however, is characterized by its ease of installation, as the vast majority does not require additional equipment beyond the already installed Wi-Fi access points, which we can find indoors for internet access purposes. The initial process of collecting data (offline phase) in the Fingerprinting method in parallel, although time consuming, is performed once during the installation of the system, and then it is necessary to repeat it only if one of the Wi-Fi access points is moved to a different location or a new one is added with a permanent presence in the space. Wi-Fi technology, although it cannot be successfully applied to custom AR applications, is the ideal method for fast navigation or guided tours of large indoor spaces of cultural 
interest (for example large museums). The managers of large museums and archeological sites have the opportunity to activate in cooperation with Apple Indoor Maps (Apple Indoor Map Program 2021), the internal location service via Wi-Fi to the users of iOS, iPadOS, while similar services are available from companies such as Here Maps for Android users, always of course with the restrictions set in the respective versions of the operating system.

The BLE protocol, like $\mathrm{Wi}-\mathrm{Fi}$, is characterized by reduced positioning accuracy. At the same time, the reliability of the measurements of the distances between fixed and mobile radio beacon is reduced, as it is greatly affected by multi-reflection errors with adjacent metal and non-metal surfaces. The accuracy level of two meters that it provides, does not make it suitable for AR applications. Characteristic advantages of the technology are the ease of installation of fixed beacons in known coordinates within the area of cultural interest, because unlike UWB technology, they do not require a constant power supply, as they can be powered by small coin cell batteries, up to 4 years. At the same time, no separate mobile beacons (Tags) are required as all the latest smart mobile devices running iOS/iPadOS and Android, have firmware, suitable to support the BLE protocol. Both fixed beacons and the rest of the equipment (Gateways) required to implement a BLE based indoor positioning system, as well as programmable tools for exporting the location of users' mobile devices, are available at a very low cost. A positioning system based on the BLE protocol could provide an appropriate tool for navigating and touring cultural heritage sites, but not the technology that would power with location data an augmented reality application.

GNSS systems are the optimal technology for outdoor localization in areas without dense and high barriers. The modern dual-frequency GNSS systems, which are enclosed in selected models of smart mobile devices of various manufacturers, provide with algorithmic procedures of absolute or relative positioning, accuracy close to 1 meter. However, this receiver technology is not currently included in all mobile devices on the market and is limited to an Android environment. At the same time the accuracy of one meter is not satisfactory enough for AR applications. On the contrary, the accuracy of a few centimeters by applying RTK techniques that we receive with the help of external low-cost dual-frequency GNSS receivers such as the u-blox ZED-F9P, which can be adapted to the body of all mobile devices of different manufacturers, is the most suitable for AR applications.

In summary, we conclude that the most suitable positioning technologies intended for AR applications are the UWB protocol for indoor and GNSS-RTK systems adaptable to mobile devices, for outdoor areas of cultural interest.

\section{ACKNOWLEDGEMENT}

This paper has been accomplished on behalf of the project SMART EYE: Development of a prototype for smart visualization of invisible antiquities. The project is running under the framework of the Operational Program Competitiveness, Entrepreneurship and Innovation 2014-2020 (EPAnEK), Special Actions "Aquaculture" - "Industrial Materials" - "Open Innovation in Culture", Т6ҮВП-00023.

\section{REFERENCES}

Haugstvedt, A.C., Krogstie, J., IEEE International Symposium on Mixed and Augmented Reality 2012 Science and Technology Proceedings 5 - 8 November 2012, Atlanta, Georgia, https://doi.org/10.1109/ISMAR.2012.6402563.
Chang, L., Niu, X., Liu, T., Tang, J., Qian, C., (2019) GNSS/INS/LiDAR-SLAM integrated navigation system based on graph optimization. Remote Sensing. 11. 1009, https://doi.org/10.3390/rs11091009.

Shih, N., (2020). Digital Preservation and Reconstruction of Old Cultural Elements in Augmented Reality (AR). Sustainability. 12. https://doi.org/10.3390/su12219262.

Merchán, M.J., Merchán, P., Hernández, E., (2021). Good Practices in the Use of Augmented Reality for the Dissemination of Architectural Heritage of Rural Areas. Applied Sciences 11. 2055. https://doi.org/10.3390/app11052055.

Boboc, R., Duguleana, M., Voinea, G., Postelnicu, C., Popovici, D.M. Carrozzino, M., (2019). Mobile Augmented Reality for Cultural Heritage: Following the Footsteps of Ovid among Different Locations in Europe. Sustainability. 11. 1167. https://doi.org/10.3390/su11041167.

Marto, Anabela \& Gonçalves, Alexandrino. (2019). Mobile AR: User Evaluation in a Cultural Heritage Context. Applied Sciences. 9. 1-14. https://doi.org/10.3390/app9245454.

Feigl, Tobias \& Porada, Andreas \& Steiner, Steve \& Löffler, Christoffer \& Mutschler, Christopher \& Philippsen, Michael (2020). Localization Limitations of ARCore, ARKit, and Hololens in Dynamic Large-scale Industry Environments. 307318. https://doi.org/10.5220/0008989903070318.

Augello, Agnese \& Infantino, Ignazio \& Pilato, Giovanni \& Vitale, G., (2021). Site Experience Enhancement and Perspective in Cultural Heritage Fruition-A Survey on New Technologies and Methodologies Based on a "Four-Pillars" Approach. Future Internet. 13. 92.

https://doi.org/10.3390/fi13040092.

Luna, Ursula \& Rivero, Pilar \& Vicent, Naiara. (2019). Augmented Reality in Heritage Apps:Current Trends in Europe. Applied Sciences. 9. 2756. https://doi.org/10.3390/app9132756.

Azzari, Pietro \& Di Stefano, Luigi \& Tombari, Federico \& Mattoccia, Stefano. (2008). Markerless Augmented Reality Using Image Mosaics. 5099. 413-420. https://doi.org/10.1007/978-3-540-69905-7_47.

Kolivand, H., A. Rhalibi, Mostafa Tajdini, S. A. Abdulazeez and Pisit Praiwattana. "Cultural Heritage in Marker-Less Augmented Reality: A Survey.” (2018). https://doi.org/10.5772/INTECHOPEN.80975.

Herbers, Patrick \& König, Markus. (2019). Indoor Localization for Augmented Reality Devices Using BIM, Point Clouds, and Template Matching. Applied Sciences. 9. 4260. https://doi.org/10.3390/app9204260.

GPS. GPS: The Global Positioning System. 2021. Available online: https://www.gps.gov/ (accessed on 18 May 2021)

Information Analytical Centre of GLONASS and GPS Controlling. 2021. Available online: https://www.glonassiac.ru/en/ (accessed on 18 May 2021).

European GNSS Service Centre|European GNSS Service Centre. 2021. Available online: https://www.gsc-europa.eu/ (accessed on 18 May 2021). 
BeiDou Navigation Satellite System. 2021. Available online: http://en.beidou.gov.cn/ (accessed on 18 May 2021).

Vasisht, D.; Kumar, S.; Katabi, D. Decimeter-level localization with a single WiFi access point. In Proceedings of the 13th USENIX Symposium on Networked Systems Design and Implementation (NSDI '16), Santa Clara, CA, USA 17-18 March 2016; pp. 165-178.

Liu, H.H.; Yang, Y.N. WiFi-based indoor positioning for multifloor environment. In Proceedings of the TENCON 20112011 IEEE Region 10 Conference, Bali, Indonesia, 21-24 November 2011; pp. 597-601.

Xiong, J. Pushing the Limits of Indoor Localization in Today's Wi-Fi Networks. Ph.D. Thesis, UCL (University College London), London, UK, 2015.

Luo, Y.; Law, C.L. Indoor positioning using UWB-IR signals in the presence of dense multipath with path overlapping. IEEE Trans. Wirel. Commun. 2012, 11, 3734-3743.

DWM1001 Battery Lifetime Estimation. 2021. Available online:

https://decaforum.decawave.com/uploads/default/original/1X/2e 9f701294239686ad22cf6e53219614483ad924.pdf/ (accessed on 18 May 2021).

Waadt, A.; Wang, S.; Kocks, C.; Burnic, A.; Xu, D.; Bruck, G.H.; Jung, P. Positioning in multiband OFDM UWB utilizing received signal strength. In Proceedings of the 2010 7th Workshop on Positioning, Navigation and Communication, Dresden, Germany, 11-12 March 2010; pp. 308-312.

Witrisal, K.; Hinteregger, S.; Kulmer, J.; Leitinger, E.; Meissner, P. High-accuracy positioning for indoor applications: RFID, UWB, 5G, and beyond. In Proceedings of the 2016 IEEE International Conference on RFID (RFID), Orlando, FL, USA, 3-5 May 2016; pp. 1-7.

Apple Indoor Map Program. 2021. Available online: https://register.apple.com/indoor/ (accessed on 18 May 2021).

Anagnostopoulos, G.G.; Deriaz, M. Accuracy enhancements in indoor localization with the weighted average technique. SensorComm 2014, 2014, 112-116.

Sklar, B. Rayleigh fading channels in mobile digital communication systems. I. Characterization. IEEE Commun. Mag. 1997, 35, 90-100.

Kaemarungsi, K. Design of Indoor Positioning Systems Based on Location Fingerprinting Technique. Ph.D. Thesis, University of Pittsburgh, Pittsburgh, PA, USA, 2005.

Jianyong, Z.; Haiyong, L.; Zili, C.; Zhaohui, L. RSSI based Bluetooth low energy indoor positioning. In Proceedings of the 2014 International Conference on Indoor Positioning and Indoor Navigation (IPIN), Busan, Korea, 27-30 October 2014; pp. 526-533.

Kanaris, L.; Kokkinis, A.; Liotta, A.; Stavrou, S. Fusing bluetooth beacon data with Wi-Fi radiomaps for improved indoor localization. Sensors 2017, 17, 812 .

Kriz, P.; Maly, F.; Kozel, T. Improving indoor localization using bluetooth low energy beacons. Mob. Inf. Syst. 2016.
Spachos, P.; Plataniotis, K. BLE Beacons in the Smart City: Applications, Challenges, and Research Opportunities. IEEE Internet Things Mag. 2020, 3, 14-18.

Corbacho Salas, A. Indoor Positioning System Based on Bluetooth Low Energy. Bachelor's Thesis, Universitat Politècnica de Catalunya, Barcelona, Spain, 2014.

Fotiou, A., Pikridas, C., 2012. GPS and Geodetic Applications. Second edn, Ziti Editions, ISBN: 978-960-456-346-3.

Rietdorf, A., Daub, C., Loef, P., 2006. Precise positioning in real- time using navigation satellites and telecommunication. Proceed- ings of the 3rd Workshop on Positioning, Navigation and Communication. 